\title{
The dynamical essence of powers
}

\author{
Andrea Roselli ${ }^{1}$ (D) Christopher Austin ${ }^{1}$
}

Received: 30 November 2020 / Accepted: 11 October 2021 / Published online: 11 November 2021

(C) The Author(s) 2021

\begin{abstract}
Powers are properties defined by what they do. The focus of the large majority of the powers literature has been mainly put on explicating the (multifaceted) results of the production of a power in certain (multifaceted) initial conditions: but all this causal complexity is bound to be-and, in fact, it has proved to be-quite difficult to handle. In this paper we take a different approach by focusing on the very activity of producing those multifaceted manifestations themselves. In this paper, we propose an original account of what the essence of a power consists in which stems from a radical reconceptualisation of power-causation according to which counterfactuals are to be explained away by powers, and not vice-versa. We call this approach the dynamical operator account of powers. According to this account, the causal role of powers consists in their ensuring that the ontological transition from a stimulus $\mathrm{S}$ to a manifestation $\mathrm{M}$ happens. Powers thus have a dynamical essence which consists in the fundamental activity of generating the counterfactuals typically associated with them. We show that if one conceptualises this functional activity as the metaphysical fulcrum around which counterfactual-based causation revolves, one is granted not only an improved methodology to individuate powers but also a better understanding of their knowability, modality and directedness.
\end{abstract}

Keywords Powers · Activity · Causation · Dynamical essence $\cdot$ Dynamical operator account

This article belongs to the topical collection on New Foundations of Dispositionalism, edited by Andrea Raimondi and Lorenzo Azzano.

The research and editorial work carried out by the co-authors toward this publication was supported by the Leverhulme Trust as part of the project Part-whole relations within the fundamental potentialities in nature (RPG-2018-079).

$\bowtie$ Andrea Roselli

roselli.uniroma3@gmail.com

Christopher Austin

christopherja@gmail.com

1 Durham University, Durham, UK 


\section{Introduction}

Recent years have seen the return and rise of power realism among metaphysicians. The same properties that once incited the ire of the reductionist paradigm prevalent in the early half of the twentieth century, are now commonplace features in the most prominent ontologies proposed by philosophers working in everything from the theory of mind to the interpretation of quantum mechanics. In the earnest effort to treat powers with ontological sincerity, contemporary characterisations of what it is to be a power have become increasingly complex. Among 'powers ontologists' it is now widely understood that even the once seemingly simple and straightforward task of providing an analysis of the philosopher's favourite powers- 'fragility', 'solubility', etc.-is in itself a rather substantial theoretical undertaking. In short, this is because elucidating the identity conditions of powers-properties essentially defined by what they do-requires an analysis capable of encompassing all the subtle intricacies of both causation and modality which coalesce in their characteristic activities.

Unfortunately, though perhaps unsurprisingly, the attempt to sufficiently address the metaphysical minutiae involved in individuating powers has resulted in the literature being plagued with an unwieldly proliferation of conceptual idiosyncrasies independently operating within a variety of distinct theories-theories which are, more often than not, entirely incommensurate (if not plainly incompatible) with one another. Indeed, in the contemporary powers literature, theories that aspire to describe what it is to be a power are underpinned by a diverse range of philosophical projects, most of which are concerned either with the creation of sui generis causal novelties or else the careful curation of ceteris paribus conditioned counterfactuals. In our estimation, however, the analysis of the identity and individuation of powers, in the vast majority of these theories, suffer from a common and crucial problem: rather than accounting for the causal-cum-modal complexity of powers, they have merely compounded it. From our perspective, in this well-intentioned attempt to transform the nomic nuances of powers into their identity conditions, something fundamental has been lost in translation-namely, the dynamical essence of powers.

In this paper, we propose a novel account of what the identity of a power consists in. In developing this account, our central guiding principle is that in any suitably robust ontological theory of powers, the causal-cum-modal complexity of their characteristic activity should be the explanandum for which the identity, or nature of powers is the explanans. Properly respecting that principle, we claim, requires a radical reconceptualisation of the 'causal role' of powers - one that is capable of dynamically differentiating the effects of powers from the essence of powers.

This paper has two main parts. In the first part, in discussing the shortcomings we see in a survey of the current "state of play" in the literature, we distil two desiderata that we believe any adequate account of the identity of powers must satisfy, which we call unity and intrinsicality. We examine a number of candidate ways to satisfy the first desideratum present in the powers literature, but find them fundamentally unsatisfactory. This is because properly satisfying unity, we argue, crucially depends upon the satisfaction of intrinsicality - and none of the main strategies for satisfying unity currently on the market are capable of doing both. In the second part of the paper, we make clear the intimate relationship between causation and intrinsicality and explore 
how this desideratum might be satisfied by various metaphysical frameworks which purport to describe in what the causal power of powers consists. After showing that none of these frameworks are sufficiently up to the task, we offer our own metaphysical schema in which a novel conception of the causal role of powers is shown to satisfy both desiderata in a coherent and compelling fashion. Finally, we compare this way of understanding the identity of powers with some other (similar, under certain respects) proposals put forward in the literature, showing the novelty of our original proposal and the need for this different approach.

\section{The causal complexity of identity}

From the perspective of a power ontology, the denizens of the natural world play an ineliminable role in establishing and directing the causal structure of the progression of its events throughout time. As a fundamentally non-Humean perspective, the constraints upon what can happen and what must happen in the world are neither contingent, nor extrinsic: they are not imbued upon it from a set of higher-order natural laws (which may have been different), nor derivable from brute facts about its causal regularities (which may not have occurred). Instead, in virtue of their operating as the ontological ground of specialised potentiality, it is the intrinsically directed natures of powers that both deliver and delimit the space of possibilities for the entities which possess them, and thereby causally underwrite the patterns of causal regularities we observe within the world.

Powers are then intrinsically dynamical properties, whose identity consists in what they do. As the standard story goes, the dynamical nature of powers consists in their being causally responsible for reliably and repeatedly bringing about a particular type of state of affairs - a 'manifestation' - upon the obtaining of a particular condition - a 'stimulus'. Thus, the identity of powers has always been closely tied to subjunctive or counterfactual conditionals of the form $<$ if this stimulus occurs, this manifestation occurs $>.{ }^{1}$ Very often, this identity was characterised by a single such counterfactual in what's come to be known as a 'simple conditional analysis' (SCA). However, although it originally served its purpose well, over time it became increasingly obvious that the SCA suffered from a variety of significant shortcomings. With the rise of ever more exotic counterexamples in the form of 'masks' (Bird, 1998; Johnston, 1992), 'finks' (Martin, 1994), and even wizards (Lewis, 1997) populating the literature, the philosophical project for the power ontologist quickly focused on searching for ways to formulate non-trivial ceteris paribus clauses with which to overcome the SCA's glaring shortcomings (Choi, 2006; Gundersen, 2002).

In the contemporary literature on power ontology, the project of 'reforming' the SCA is much less prominent than it once was. In the first place, philosophers who

\footnotetext{
1 Throughout this paper we will make implicit use of the standard semantics for counterfactual conditionals, where they are construed as 'variably strict conditionals' which extensionally quantify over possible worlds, as developed by Stalnaker (1968) and Lewis (1973). We will not here address the many and varied interpretive issues concerning the possible worlds framework that have been raised by powers ontologists, as the dialectic of this paper is largely independent of the stance one takes on them.
} 
are realists about powers have disassociated themselves from any analyses of powers which would reduce them to counterfactuals. However, given that the identity of powers - even on a realist view of powers-is often captured by a counterfactual, understood now as the relevant conditional which its causal-cum-modal nature metaphysically underwrites/makes-true, this project remains important. Why then has it been (largely) halted? We suspect that there is a straightforward, but important reason why this is the case. Once powers had emerged from their being treated with positivist suspicion - that is, as merely heuristic artefacts, ideally to be explained away (Mellor, 1974) by a process of eliminative analysis - and began to be accepted as genuinely robust causal actors in the natural world, it became clear that the implicit complexity of the counterfactuals in the SCA only scratched the surface of the complex nature of these properties. Outside of the idealised confines of the thought-experiment laboratory, what these properties do and how they do it is no simple matter. Accordingly, for any philosopher who wished to treat powers with ontological sincerity, it became obvious that the identity of a power-that which specifies its causal-cummodal nature-cannot be captured by a single conditional.

There are two complimentary conceptual sources for this realisation that we think are important which we will highlight here. The first concerns the antecedent of the conditionals which characterise powers, and it is what we call the polygeny of powers: the stimulus conditions of powers are necessarily multifaceted. Arising at least partly from powers theorists' attempt to recover the force of necessity carried by the counterfactual connective $(\square \rightarrow$ ) in light of 'masking' phenomena, it has been recognised that the characterisation of the stimulus condition of a power as being comprised of a single causal factor is misguided. Consider, for instance, a relatively simple power-the 'flammability' of a match. At first pass, the stimulus condition for this power is simple and singular - the striking of the match. However, as Goodman (1979) noted some time ago, it's easy to see that 'striking' is not the only relevant causal factor in the match's ignition: without the appropriate Oxygen presence, the suitable roughness of the matchbox, the relatively low wind levels, etc., no amount of 'striking' is going to lead to the match's 'flammability' being manifested. ${ }^{2}$

It can hardly be denied that these considerations are going to be generalisable for any account of the causal role of powers, and thus that the appropriate stimulus condition for any particular power will necessarily be comprised of a number of distinct causal factors being in place. ${ }^{3}$ The complexity doesn't stop here of course, as each of the causal factors that must be included in a single power's stimulus condition are themselves independently variable and capable of taking on multiple distinct values-e.g.

\footnotetext{
2 Another way of putting this point is to say that the causal relation between stimulus and manifestation in powers is non-monotonic - that is, that the truth of the conditional < if struck, then ignite $>$ is not necessarily preserved under "strengthenings of the antecedent" in the form of the addition of other causal factors-see Schrenk (2010).

3 Of course, the determining the extent of the relevant scope of a power's 'stimulus condition' is fraught with conceptual difficulties. On the one hand, attempting to establish a membership criteria that discerns between relevant 'causal' factors and mere 'background conditions' is likely to be a largely ad hoc enterprise (Mumford and Anjum 2011). On the other hand, however, including every causally relevant factor in a power's stimulus condition might require including world-encompassing 'totality facts' (Armstrong 1997; Jacobs, 2011) and so, to ensure causal 'closure', perhaps even absences (Hauska 2009) in the intrinsic essence of a power; for a good overview of the ontological oddity of totality facts, see Keller (2007).
} 
more or less Oxygen, more or less wind, etc. And so the stimulus condition of a single power must comprise not merely a set of causal factors, but a "second-order" set comprised of all of the quantitatively distinct value-variations of the members of that initial set of factors which lead to that power's manifestation occurring (Manley \& Wasserman, 2008; Vetter, 2015). This multifaceted complexity inherent in the stimulus condition of a power is what the claim that powers are polygenic consists in.

With the polygenic nature of powers in mind, the next conceptual source for the complexity of a power's causal-cum-modal nature can be easily understood: it is in many ways the mirror of polygeny which concerns the consequents of the conditionals that characterise powers, which we call pleiotropy. ${ }^{4}$ It has been widely acknowledged that, owing to the polygenic nature of their stimulus conditions, the manifestations of powers are also similarly multifaceted. For a simple illustration, consider again our example of the flammability of the match. Pleiotropy reflects the fact that, with all of the possible value-variation complexity in the stimulus of this power (changes in the amount of Oxygen, in the wind levels, etc.) necessarily comes a range of possible quantitative and qualitative value-variation complexity in the manifestation of the power: more Oxygen results in caustic ignitions, greater wind results in weaker flames, etc. In the literature, this aspect of powers is often referred to-following Ryle (1949) — as their being 'multi-track', where single 'tracks' are conceptualised as the combination of a power's second-order set of stimulus factors with specific values and a corresponding particular value of the power's associated manifestation-state (Ellis, 2001; Martin, 2007; Vetter, 2013; Williams, 2011). ${ }^{5}$

As it turns out then, treating powers not merely as descriptively convenient referents of collections of reductively primitive categorical properties, but as robust and causally capable constituents in one's ontology, requires some amount of conceptual subtlety, as specifying the causal-cum-modal nature of a power, in which its identity consists, is a rather complicated affair: on both sides of the conditional, which is taken to capture that identity, comes complexity. Properly taking the complexity exhibited in the combination of polygeny and pleiotropy into account means that for the powers ontologist, even the most ordinary, seemingly simple power must become, as Vetter (2013: p. 346) puts it, "a beast with infinitely many heads". In other words, according to the framework in which powers are understood, characterising the identity of a power requires - to put it most mildly-more than one conditional. More specifically, it requires a set of conditionals where various S-values (each of which represent sets

\footnotetext{
4 This term, like polygeny, originates from the field of developmental genetics, where it refers to the phenomenon of a single genetic locus exerting causal influence upon multiple distinct phenotypic traits. Molnar (2003: pp. 194-195) utilises both terms in his account of powers, though he takes the pleiotropy of powers to refer to the phenomenon of a single power's manifestation contributing to multiple distinct effects, which are diachronically downstream from and not identical to manifestations. Our use is of course more "direct" and powers-focused, and so the additional metaphysical machinery of Molnar's account-where manifestations are conceptualised as 'contributions' to effects-wont' be appealed to here.

5 For the moment, we are taking 'multi-track powers' to refer only to the phenomena of a power's manifestation state being quantitatively variable. There are of course many philosophers who endorse qualitative multi-tracking, and hold that a single power can produce a variety of qualitatively distinct manifestation states (Heil 2003; Martin 2007; Williams 2011). In general, because we judge qualitative multi-tracking to be untenable, and because it is much more contentious than quantitative multi-tracking in the literature, we leave it out of our main discussion; for a prominent critique see (Lowe, 2010).
} 
Fig. 1 a a collection of causal factors $\left(\mathrm{S}_{\mathrm{a}}-\mathrm{S}_{\mathrm{f}}\right)$ in particular states (a-2, b-1, etc.) which constitute a single 'stimulus' condition in the antecedent of a single counterfactual in $\mathbf{b}$ the set of counterfactuals which characterise the multifaceted causal activity of a power

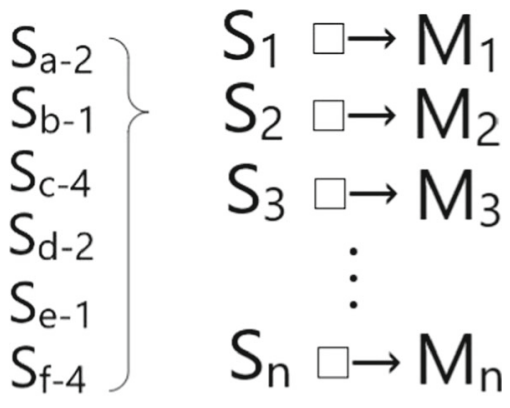

(b)

of causal factors in specific states, as discussed above) are correlated with various M-values (each of which represent a specific quantitative or qualitative degree of a power's manifestation state); see Fig. 1 below.

With all of this complexity in mind, let us return to our initial question: in what does the essence of a power consist? In the contemporary literature, the ongoing attempt to capture the multifaceted nature of the characteristic causal role of a power has culminated in the conception that what it is to be a power is exhausted by an exceedingly long (perhaps infinite) complex collection of counterfactual conditionals. While we do not wish to downplay the successful and important work of theorists who have carefully dissected the metaphysical structure of powers in light of their causal complexity, in our estimation the picture of the identity of powers this sort of analysis is apt to deliver is fundamentally problematic.

There are two issues that we want to particularly highlight here. The first is that, on this sort of picture, the set of counterfactuals with which the essence of a power is identified is comprised of necessarily discrete and fundamentally disjoint 'causal roles' that power is capable of performing. This picture thus fails to offer any insight whatsoever as to why or how it is that all of these exceedingly specific, non-overlapping causal roles collectively belong to, or metaphysically 'flow from' the self-same and singular essence of any particular power - call this the unity problem. The second issue with this picture we want to raise is that, as an artefact of its conceptual structure, it is a model capable of capturing the multifaceted causal complexity at the centre of a power only from an 'outside', or extrinsic perspective-that is, via its effects (as represented by the various counterfactuals which belong to its associated set). Accordingly, the account of the identity of a power on this picture necessarily equates the extrinsic, epistemologically accessible activities of a power with its intrinsic dynamical nature, and is thus fundamentally incapable of elucidating the way in which the latter produces or otherwise metaphysically generates the causal structure of the former-call this the intrinsicality problem. $^{6}$

We believe that both the unity and intrinsicality problem highlight serious conceptual deficiencies in the metaphysical framework with which contemporary accounts of

\footnotetext{
6 Although conceptually distinct, the metaphysical concerns of this second issue are clearly closely related to those of the first. Throughout this paper, we will touch on their similarities and overlaps where appropriate.
} 
the identity of powers are constructed. With all of the aforementioned causal intricacies implicit in any sufficient analysis of the essence of powers in mind, we therefore propose that any suitably robust account of power-identity must be one that is explanatory with respect to the manner in which the polygenically and pleiotropically complex activities of a power are integrated by and metaphysically grounded in its intrinsic nature. That is to say, in other words, that any suitably robust account of power-identity will be one in which the conceptual challenges represented by unity and intrinsicality are sufficiently met; in this article, we suggest a promising way towards this direction.

\section{On the origin of ontological unity}

Let us first examine the question of unity: in what way are the vast and disparate causal roles a power is capable of performing metaphysically united as various "expressions of" its (singular) essence? Though this question has not gone unnoticed in the powers literature, it has certainly yet to receive the serious attention we believe it deserves. In fact, a rather prominent strategy employed in the literature consists in simply dissolving, rather than solving, the 'problem of unity'. To that end, many philosophers who are themselves ardent promoters of a powers ontology have argued that if there is any unity to be found among the multiple causal roles (and associated counterfactuals) which we attribute to a single power, it is an accord born of linguistic, or heuristic convention and is thus fundamentally epistemological, rather than ontological in nature.

The central claim in this line of thought is that, ontologically, there is a one-one correspondence between counterfactual and power-or, to put it another way, that only 'single-track powers' exist (Bird, 2007; Lowe, 2006, 2010). One popular way of cashing-out this claim is via the property-predicate distinction (Ellis, 2001). 'Fragility' (for instance), which we might normally conceptualise ontologically as a power that plays a multiplicity of distinct causal roles, might instead be understood as being merely a predicate whose extension ranges over a particular collection of fundamentally discrete and separate properties - that is, the various single-track powers of the form $<$ if struck with this force at this ambient temperature, crack in this way $>$ and $<$ if struck with that force at that ambient temperature, break in this way $>$, etc. According to this perspective, which Vetter (2013) calls austere dispositionalism, the phenomenon of a set of discrete causal roles 'flowing from' the essence of, and thus collectively belonging to a single power is only an artefact of descriptive convenience. And so clearly if one adopts this sort of view, there is no problem of unity which stands in need of solving: while there may yet remain semantic 'one-many'-type issues with respect to reference, meaning, etc. when dealing with the multiple causal roles attributable to a single power, these have no proper ontological correlates.

While cutting this knot-rather than untying it - is certainly an available option, we judge this to be an unproductive way forward, for two reasons. Firstly, adopting this view leads to a highly unparsimonious ontology of powers. Because on this view every fine-grained variation in the collection of stimuli factors and manifestation-states typically associated with the activity of a single power are instead each individually correlated with a numerically distinct power, the resultant ontological inventory of the 
powers theorist becomes infinitely inflated. In fact, if one adopts this view, the inflation caused by endorsing a trope-theoretic view of powers (according to which, for instance, every object susceptible to being broken has its own, particularised power of 'fragility') is just the tip of the iceberg: every such power-trope will in fact be composed of a non-denumerably vast set of 'tropettes', one for every possible unique combination of stimulus and manifestation state-values (or, one for every unique counterfactual, as in Fig. 1b). The defender of this view can of course give the well-worn Lewisian (1973) reply that theirs is the acceptable form of parsimony (that is, quantitative, rather than qualitative), but this remains a cost of the view as it will no doubt strike many (as it does us) to be an unnecessary proliferation of highly unnatural properties.

Secondly, and more importantly, the semantics of our linguistic practices constitute an explanatorily insufficient framework with which to capture the metaphysics of unity. In short this is because we believe that the fact that certain sets of properties are consistently found to be "bundled" together with one another-what is often called the phenomenon of clustering — stands in need of an ontological, rather than an epistemological explanation. ${ }^{7}$ Outside of adopting an Idealist perspective on our role in constructing the ontological landscape, an appeal to the heuristics of reference leaves the clustering of causal roles associated with a single power utterly inscrutable. Why should it be the case, for instance, that certain collections of the aforementioned 'tropettes' concerning "breaking when stuck" are all regularly found together in vases? Without a firm ontological grounding of the phenomenon of clustering, it signifies, as Lowe (2006: p. 136) put it, the "existence of enormous and mysterious cosmic coincidences as a matter of brute, inexplicable fact". 8

In order to avoid an embarrassment of riches and intractable bookkeeping then, we suggest that the powers theorist ought to treat the unity question with ontological sincerity. The important question to ask is: what is it about a power that, metaphysically, accounts for the fact that the various particular causal roles it is capable of performing "flow from" its nature in such a way that they all collectively "belong to" it? Unfortunately, direct answers to this question are few and far between in the contemporary powers literature. There are however at least two strategies for answering the unity question which have recently been pursued that merit examination here.

The first is what we call the unity viaexemplification strategy, according to which the various causal roles (and their associated counterfactuals) which are associated with a single power are united by their being exemplifications, or ontological "instances" of that power. ${ }^{9}$ The idea here is akin to treating these causal roles as determinates of a single determinable: for instance, $<$ if struck with this force, will break in this way $>$ might be understood as being a determinate causal role of the more generalised $<$ if

\footnotetext{
7 The phenomenon of 'clustering' has been discussed in a variety of other philosophical contexts, some of which don't directly concern a powers ontology in any way. For instance, a prevalent view of species-identity in contemporary philosophy of biology casts the 'essence' of a species as a special kind of 'homeostatic property cluster' - see Boyd (1999), and Wilson et al., (2007).

8 For other powers-based discussions of the phenomenon of clustering, see Williams (2011; 2019), Lowe (2006), Mumford (2004), Chakravartty (2007), Oderberg (2011), and Dumsday (2019).

9 Note here that as entire causal roles or counterfactuals are in view, we do not (and should not) refer to these as the various 'manifestations' of a power-a term reserved for the effects/consequents of those roles/counterfactuals.
} 
struck, will break > role which represents the essence of 'fragility'. The sort of unity a collection of determinates under a single determinable enjoy might be an appropriate fit for the kinds of cases we're considering, as it is arguably not merely linguistic, but plausibly reflects ontology_it's not simply due to semantic convention, for instance, that 'scarlet', 'crimson', etc. are all "unified under" the determinable 'red'. Pursuing this sort of strategy, one might claim that the various causal roles reflected in the polygenic and pleiotropic nature of 'multi-track' powers-each of which is captured by the combination of a highly specified set of S-values and a particular M-value-are just fine-grained, determinate exemplifications of the primary causal role of the power, the more coarse-grained, determinable causal role captured by $<\mathrm{S} \square \rightarrow \mathrm{M}>.{ }^{10}$ On this perspective, the sense in which the diverse collection of causal roles (and their accompanying counterfactuals) associated with a particular power are one is clear: as (limited) expressions of a single power, these diverse causal roles are unified in their being variations on or permutations of a single causal "theme", so to speak. ${ }^{11}$

Although the 'unity via exemplification' strategy appears promising, we think that the determinate/determinable distinction at its core engenders substantial problems in the context of an ontology of powers. Firstly, the way in which the various causal roles of a power are "present" in it does not seem to be capturable by the implicitly exclusionary way in which determinates are "present" in a determinable. While it's true that a single power can only manifest in one specific way at any particular time, it's not the case that the various causal roles which "belong" to it (each of which correspond to a specific manifestation) have the same limitation-they are each "present" in that power at all times in the same way, irrespective of whether they are currently "on display". To put the point in another, more familiar way: a power makes-true all of the counterfactuals which represent its various causal roles no matter which or whether their antecedents are satisfied at any particular time, or in any particular instance. Importantly then, this fact about the nature of powers stands in stark contrast with the nature of the determinate/determinable relation according to which having multiple determinates of a single determinable at any one time is impossible. ${ }^{12}$

Secondly, the unity via exemplification strategy does not deliver an ontologically robust sort of unity. For although the unity of various determinates under a single determinable is suitably mind-independent (insofar as it is not merely linguistic, or a matter of convention), it is not necessarily ontologically significant. "Unity under a determinable" is the sort of unity which is enjoyed by, for instance, the set of all the

\footnotetext{
10 We note in passing here that the 'unity via exemplification' strategy does not look especially promising for those who conceptualise powers as being not only quantitatively, but also qualitatively multi-track: while the sense in which quantitatively distinct determinates belong to a single determinable is clear, the same cannot be said — or at least, cannot be said as perspicuously — for qualitatively distinct determinates. See footnote 5 .

11 The analogy between 'exemplification' and 'determinate' in the case of powers is of course not wholly precise, given that determinates_rather than determinables - are typically understood as being ontologically primary. That said, there may be at least some cases in which determinables ought to be understood as more ontologically fundamental than their determinates—see Wilson (2012).

12 In her discussion of the ontology of multi-track and single-track powers, Vetter (2015: p. 53) makes this point in passing: "Having a determinable property entails having one of its determinates, to the exclusion of all others. Having the multi-track disposition electric charge, on the contrary, entails having all the corresponding single-track dispositions".
} 
quarks and electrons in the universe under the determinable 'particle' - and this is, though not entirely ephemeral, a rather ontologically weak form of unity. This sort of unity thus doesn't strike us as having the ontological "strength" to best capture the way in which a vast and disparate collection of causal roles "belong to" a single power: that all of the counterfactuals which represent those roles are not "entirely loose and separate" (as Hume would have put it) is a fact that doesn't seem to be secured in virtue of their being a set of determinates which fall under a single determinable.

The second strategy for unification which has been explored in the literature is what we call the primitive unity strategy, according to which the various causal roles (and their associated counterfactuals) which are associated with a single power are united as a matter of conceptual necessity. The motivating thought here is the denial that there is any metaphysically rich "bundling" relation that holds either among the multiple causal roles of a power or between those roles and a power's more fundamental nature that unites or ontologically ties them together (Mumford, 2004). ${ }^{13}$ Rather than positing that the various causal roles of a power point to something more ontologically, or causally primary about that power, this strategy grants primacy to those roles themselves: the set of those roles just is the essence of that power. In virtue of what then are these multiple causal roles in some way one? The answer, according to this strategy, is that these roles are one for just the same reason, and in just the same way as the multiple members of a set are one-that is, via 'concept-containment': the nature of a power-like a set-is, as Mumford (2004: p. 173) puts it, "exhausted by its extension", and so the 'clustering' together of the various causal roles in a single power is explained by the simple fact that their doing so is the existential sine qua non of that power; and thus, in at least a Kripkean 'weak' sense of necessity, they could not have possibly failed to do so, on pain of that (particular) power failing to exist. ${ }^{14}$

Because it makes a principal appeal to the necessity of set-membership, this strategy at first glance appears to invoke a type of unity that is stronger, or more ontologically robust than the one at the core of the unity via exemplification strategy. However, we think that closer inspection reveals it to be rather weak in a significant respect, as it is incapable of delivering any principled way in which to either restrict or license the ontological reduction and inflation of powers. To see why this is a problem, let us suppose that collective set-membership is the correct model for capturing the unity that holds among the members of a 'cluster' of causal roles in virtue of which they constitute a single power. Because the principles of set theory don't prescribe any constraints on set construction, and thus allow arbitrary assignments of 'least/minimal upper bounds', the powers theorist is confronted with two prima facie undesirable results.

\footnotetext{
13 Employing slightly different terminology, Mumford (2004: p. 173) asks: "If there are properties that are clusters of distinct powers, however, what is the bundling relation that ties the powers together and makes them all powers of the same property or trope? In short, there is none. There is not an extra bundling element, or external relation, in addition to the powers".

14 Continuing from the quote in the previous footnote, Mumford (2004: p. 173) says that this account of unity provides "...the answer we would give for any set, in set theory, that is exhausted by its extension. There is not a tie connecting the members or binding them into the set. I propose a similar treatment for properties qua clusters. There is no extra element holding a causal power within the cluster or tying the individual powers together. But were one of the powers absent from the cluster, we would have a different cluster so different property".
} 
On the one hand, the possibility of upward and outward ontological inflation: the upward construction of increasingly complex powers out of sets of simple counterfactuals will result in mereologically 'mega-powers' - e.g. the single power composed of my ability to fight off infections, my ability to digest food, and my ability to sing,- - and the outward combination of counterfactuals concerning "distant" objects will result in mereologically expansive powers-e.g. the single power composed of my ability to sing and Big Ben's ability to chime. ${ }^{15}$ Inversely, on the other hand, the possibility of downward and inward reduction: single powers can be divided downward into multiple smaller sets corresponding to discrete groupings of counterfactuals-e.g. my power to sing into the sets of causal roles which constitute my ability to open my mouth and those that constitute my ability to vibrate my vocal cords-and single powers can be contracted inwardly into more restrictive sets of counterfactuals-e.g. a cell's power to produce energy into the set of causal roles which constitute a mitochondria's ability to perform oxidative phosphorylation.

Of course, allowing some instances of ontological inflation and reduction in particular cases is theoretically advantageous - especially when empirical evidence indicates their suitability. With that said, the crux of the issue for the powers theorist here is not that this putative principle of unity allows some of those instances, but rather that it doesn't disallow any, given that the principles of set theory permit (or at any rate, do not prohibit) the 'unrestricted composition' of elements into sets. We acknowledge that those who otherwise favour unrestricted composition will likely view the endorsement of the causal role/counterfactual-based equivalent of 'mereological universalism' here as an advantage, rather than a liability of the account. ${ }^{16}$ At the same time however, we wish to highlight the fact that, as is well known in its more straightforward and familiar mereological application, those who endorse 'unrestricted composition' in the powers case currently under discussion are saddled with the particularly uncomfortable entailment that there is no ontological distinction between odd and ordinary, or unnatural and natural powers. Even if the powers theorist is willing to forgo her commitment to a typically 'sparse' ontology, we think she should be much more reticent to endorse the claim that the metaphysical unity enjoyed by, for instance, $<$ a vase's power to break if struck and a virus' power to replicate if uncoated $>$ and that enjoyed by < a vase's power to crack if lightly struck and that vase's power to shatter if forcefully struck $>$ are ontologically on par. If this is the sort of position that the powers theorist's set-theoretic 'principle of unity' demands she adopt, we suggest that principle ought to be rejected.

\footnotetext{
15 In his critical discussion of multi-track powers, Lowe (2010: pp. 11-12) makes a point related to the upward threat, asking "...it becomes unclear why we should think that a single object may have many different powers rather than just one - a power to do all the things that it can do. And that would render the notion of power a rather feeble and trivial one".

16 For discussions of mereological universalism outside of the context of a powers ontology, see Lewis (1986), Van Inwagen (1990), and Cotnoir and Baxter (2014).
} 


\section{On the powerfulness of powers}

As far as we're concerned, for the powers theorist who wishes to take the unity problem seriously - that is, by not aiming to merely dissolve, but rather solve it - the strategies employed to address that problem present in the literature are by and large unpromising. In our view, there is a central and important reason that both of these strategies deliver the wrong results: they are primarily focused on what powers can do rather than how they do what they can do. Both of these strategies take as their conceptual starting point the effects of a power - that is, its various manifestations and correlated stimuli-and subsequently attempt to derive from them the metaphysical principles according to which they all collectively "belong" to that power.

We believe that an answer to the question of unity whose metaphysical analysis is primarily focused on a power's effects can only ever be fruitless. In general, if there is any unity among effects, it is only a derivative unity which is grounded in the nature of the cause - and in this case, the same holds true: the unity of the effects of a power are grounded in the causal-cum-modal nature of the power itself. Accordingly, we claim that any successful answer to the question of unity will be one that makes appeal to this metaphysically “deeper" source by elucidating the way in which the unity of a power's effects (its characteristic causal roles, as represented by the various counterfactuals it makes-true) is a product of how those effects are generated by its intrinsic causal activity. Strategies of the sort thus far examined are unfruitful, we believe, precisely because they do not derive unity from the powerfulness of powers, and so do not ground that unity in the dynamical essence of powers.

As explicating precisely what the 'powerfulness' of powers consists in has long been a tricky issue for the powers theorist, this is a task which is certainly more easily said than done. But for the sort of account of the identity of powers that is currently prominent in the literature which we have been examining thus far, that task is considerably more difficult. This is because, we claim, these accounts fall prey to what we earlier called the 'intrinsicality problem': in conflating a set of counterfactuals with the essence of a power, such accounts are inherently not well-suited to deliver a conception of powers as properties with an intrinsically causal nature. Indeed, we find that on these sorts of accounts, the powers theorists' pronouncement that powers are the "driving force in a causal relation" (Ellis, 2002: p. 65) rings rather hollow. This is because, to our minds, adopting an analysis of 'causality' via counterfactuals places all the power "outside of" powers.

However far away contemporary powers metaphysics may now be from its modern reductionistic and verificationist roots, it yet quite prominently retains a key conceptual component of their Humean framework-the analysis of what a power can do by means of the counterfactuals it makes-true. Given that cashing-out what the 'causal role' of a power consists in still requires the utilisation of counterfactuals (as we illustrated in the first section of this paper), a counterfactual-based analysis of causation remains at the core of contemporary powers metaphysics. Of course, while it has arguably been to date the most successful of its kind in the metaphysics literature, the counterfactual analysis of causation has had its share of conceptual difficulties over the years. For the sake of the present discussion however, let us assume that all of the problematic aspects of the analysis are in principle solvable, as our concern here is 
not whether the counterfactual analysis is capable of capturing 'causality' per se, but whether it is capable of capturing what's causal about causal powers.

In the most extensively studied and widely endorsed accounts of causation which utilise a counterfactual-based analysis, dependence and difference-making are the key concepts: for any given phenomenon with various candidate causes, the cause (or the set of causes) is the one (a) whose occurrence is the sine qua non of the effect's occurrence, and (b) whose value-variation makes a difference to the value-variation of the effect. ${ }^{17}$ Applying these concepts in the framework of a powers metaphysic then, let us ask: in what way are powers causally powerful? Consider again the philosopher's favourite power - 'fragility'. The factor upon which the occurrence of the effect (read: the manifestation of the power-i.e. 'breaking') depends is surely the stimulus-even when the power is present, without the 'striking' taking place, no effect can or will occur. Furthermore, in this causal set-up, it is variations on the value of 'striking' (striking with more or less force, etc.) that are correlated with variations on the value of 'breaking' - it is the factor which makes a difference with respect to the effect.

What is clear then is that, when utilising a counterfactual-based analysis of causation in the framework of a powers metaphysic, the power seems rather powerless: the relevant effects neither depend upon nor are made different by the activity of powers. Indeed, on this analysis, all of the relevant causation is happening "outside of" powers: it is the extrinsic stimuli of powers, and not the intrinsic nature of powers themselves, which appear to have all the causal power. Thus if the identity of a power is, as we claim that the large majority of contemporary accounts has it, exhausted by a specific set of counterfactuals, powers appear to be in the end little more than causally inert metaphysical middle-men. This is important because, as we outlined above, the impotency of powers in such accounts leads rather naturally to their inability to provide a satisfying answer to the unity question: in appealing to what powers can do rather than how they do what they can do, these accounts are fundamentally unable to elucidate the way in which the unity of a power's characteristic causal roles is a product of its intrinsic causal nature.

With the problems of (a) intrinsicality and (b) unity in mind, we claim that a philosophically sufficient and conceptually robust account of the identity or essence of a power must be one that (a) shows how its extrinsic counterfactual complexity is grounded in its intrinsic nature in such a way that (b) its set of polygenically and pleiotropically complex counterfactuals all "belong to" that power. In the next section, we will offer a new account of the essence of powers that we think is capable of meeting both criteria - the dynamical operator account.

\section{A new account of the essence of powers}

We have claimed that the central problem with contemporary accounts of poweridentity centre around the concept of power-causation: the current view of how powers can do what they do is unable to provide a satisfying account of unity and

17 For the canonical formulations of dependence and difference-making in causation, see Lewis (1973) and Woodward (2003). A more comprehensive overview of these topics can be found in the entries in Beebee et al. (2009). 
intrinsicality because on that view, powers are essentially powerless. As far as we're concerned, meeting those important challenges concerning power-identity requires a radical reconceptualisation of power-causation —one in which counterfactuals are the explananda, not the explanantia.

In recent years one of the most popular responses to the 'powerlessness of powers' on behalf of the powers theorists has been to straightforwardly reject the key role of dependence and difference-making in causation and to claim that the causal power of powers is best captured by a novel, sui generis form of causation. Attempting to conceptually distance themselves from what they see as an account of causation too fundamentally entwined with its own Humean origins, powers theorists have highlighted the various well-known problems with counterfactual-based theories of causation (pre-emption, transitivity, and overdetermination, to name but a few) and latched-on to alternative forms of causation of the sort prominently highlighted by Hall (2004), who distinguished 'productive' forms of causation from the standard 'dependence' forms: the latter are said to merely 'track the symptoms' of causation, while the former purportedly provide the "objective physical connections which Hume sought in vain" (Salmon, 1994: p. 297). ${ }^{18}$ Several variants of 'production'-based causation utilised in exclusively power-based causation are by now well-known in the contemporary literature - the tendencies of 'dispositional modality' (Mumford \& Anjum, 2011) and 'mutual manifestations' (Heil \& Martin, 1999; Martin, 2007) for instance.

As far as we're concerned these alternative accounts of causation do not offer substantial improvement over their competitor: although they often claim that counterfactual accounts are too "surface-level" and make the workings of the causal relation fundamentally mysterious, with their having jettisoned the cornerstones of dependence and difference-making, a tu quoque is readily apparent (Froeyman, 2012; Glynn, 2013; Psillos, 2004). We will not be making that argument here however, and for a principled reason: we think that the general approach that most powers theorists have taken in simply rejecting counterfactual-based causation in an attempt to restore the causal power of powers is a mistake, and we want to offer a new way forward. As we see it, it's not the core components of the contemporary concept of causation-'dependence' and 'difference-making' — which are per se problematic for the powers metaphysic, but rather the manner in which they are typically understood to be applied, or utilised therein.

In the standard application, as we have seen, stimulus factors are taken to be the most central causal element because they make a difference to whether and in which respect the manifestation of a power occurs. While we hold that dependence and differencemaking are the constitutive, or core causal notions and do not wish to deny that stimulus factors play these important roles, we claim that they do so only derivatively and thus non-fundamentally. This is because, we argue, the dependence and difference-making capabilities of stimulus factors are themselves inherited from the nature of powers. ${ }^{19}$ To see why this is so, let's return to our earlier example of the manifestation of a match's power of 'flammability'. When we discussed this example in the context of polygeny

\footnotetext{
18 Cf. Glennan (1996).

19 One of us has argued this very point about the causal nature of powers in a different context—see Austin (2015; 2018).
} 
and pleiotropy, we highlighted the fact that not only must a variety of stimulus factors and causal conditions obtain in order for that manifestation to come about-sufficient 'striking', appropriate 'oxygen levels', etc.-but that those factors' value-variations are correlated in a fine-grained fashion with the value of that manifestation which occurs in any instance. Together, these facts are certainly sufficient for stimulus factors to be of causal import with respect to a power's manifestation-but importantly, these factors are only capable of playing this crucial role in the context of a power being present.

Consider the situation in which the power of 'flammability' were not present. Would the presence of 'striking', appropriate 'oxygen levels', etc. bring about 'ignition'? Clearly not. In this situation, the various fine-grained ways in which 'striking', etc. occur would not be correlated with corresponding fine-grained changes in the way 'ignition' occurs. These factors only bear the causal relation to manifestation-events in the context of the power of 'flammability'. Thus, the dependence of 'ignition' upon the presence and state of these factors itself depends upon the presence of a power. Furthermore, even when 'flammability' is present, and 'ignition' does depend upon those factors, the particular ways in which those factors make a difference is itself ultimately dependent upon that power. Anyone who has, for instance, lit a match both from a paper matchbook and a proper matchbox knows that the same degree of 'striking' will not engender the same level of 'ignition' in both cases. The fact that there is a different instance of 'flammability' in these cases illustrates that the ultimate difference-maker- the one which dictates in which way other causally relevant factors make a difference-is the power itself. ${ }^{20}$

We believe that these simple and rather mundane facts ultimately illustrate an important point with respect to powers and their causal role: what's causal about causal powers isn't their producing a manifestation state per se, but rather, their working as a functional mediator between inputs (stimulus factors) and outputs (manifestation states) in a specific fashion. Because according to this view the essence of a power consists in the particular way in which it performs this functional activity (of assigning state-to-state values), we call this the dynamical operator account of powers. To be more precise, on our account what it is to be a particular power is in fact the very functional activity: strictly speaking, the performance of a specialised causal role is not something a power merely does, because that doing is what that power is. Thus our account, as we earlier emphasised, locates the essence of a power not in what that power can do, but rather in how that power does what it can do. ${ }^{21}$

\footnotetext{
20 Clearly, in this case, the difference between the two matches has to do with their tips. If the same striking occurs in both instances and different results are generated, our claim is that this illustrates that this is a consequence of their respective intrinsic properties-i.e. of the 'flammability' inherent in the respective chemical composition of their tips. Thus because on our view the essence of powers consists in the performance of the intrinsic activity of metaphysically ensuring that the ontological transition from stimulus to manifestation occurs, and occurs in a specific fashion, powers cannot be-as we earlier claimed our competitor accounts have it-merely "causally inert middle-men".

21 The mention of powers having a causal role which is functional might recall in some readers' minds the well-known functionalist accounts of powers-e.g. Mumford's (1999) account. This is an association we wish to distance ourselves from: not only are such accounts non-realist (and so at odds with the spirit of our account and the majority of contemporary powers ontology) but the sense in which powers are 'functional' is entirely distinct from the sense employed here. According to a functionalist account, powers-much like
} 
We acknowledge that this account presents a radical and unorthodox view of the nature of powers, but we believe that its significant conceptual benefits far outweigh what some will see as the cost associated with its unfamiliarity-most pertinently with respect to the dialectic of this paper: its ability to satisfy the desiderata of unity and intrinsicality. Consider the latter: as we earlier argued, contemporary accounts of powers, as an artefact of their conceptual structure, propose models of powers which are capable of capturing the multifaceted causal complexity at the centre of a power only from an "outside", or extrinsic perspective- that is, via its effects (as represented by the various counterfactuals which are associated with it). In conflating a set of counterfactuals with the essence of a power, these accounts are inherently not well-suited to deliver a conception of powers as properties with an intrinsically causal nature. For, on the most prominent analysis of 'causality' via counterfactuals, all of the relevant 'power' is seemingly located "outside of" powers themselves. On our account however, all of the causal work associated with powers is rooted in the unique activity that the powers themselves perform — namely, their functioning as dynamical operators which metaphysically map and diachronically translate sets of stimulus factors into the production of specific manifestation states. This intrinsic activity functions as the central metaphysical fulcrum of counterfactual-based causation and the differencemaker upon which making a difference itself depends.

Earlier we stated that the most promising strategy for properly satisfying the other desiderata-unity - crucially depends upon the satisfaction of intrinsicality. On the dynamical operator account of powers, the unity of a power's effects (its characteristic causal roles, as represented by the various counterfactuals it makes-true) is a product of the way in which those effects are generated by its intrinsic causal activity. In short, the unity of these effects is a unity of ground in that all of the available variety of polygenic and pleiotropic counterfactuals associated with a particular power are merely differential expressions of its dynamical nature (as explicated above). Previous attempts to account for unity have, as we earlier illustrated, primarily focused on the effects of powers-e.g. accounting for the phenomenon of 'clustering' via mereological relations among those effects, or via those effects being the collectively conceptually necessary members of a single set. In our view, irrespective of those specific accounts, this strategy is in general flawed: it represents an approach akin to attempting to elucidate the unity that the phases of water (i.e. gas, liquid, and solid) possess via treating them as discrete and separable individuals, rather than via their being various limited dynamical expressions of a single ground-that is, the chemical catalysation activity of the molecular constitution of water $\left(\mathrm{H}_{2} \mathrm{O}\right)$. Similarly, in line with our view of the central causal role of powers, on our account the multiple counterfactuals associated with a single power are one in virtue of their representing specific pairs of input/output values which are functional derivatives of the causal translation activity of a single operator.

\section{Footnote 21 continued}

'mental-states' in the functionalism in the philosophy of mind from which these accounts are drawn-are little more than descriptive devices meant to gesture toward the activities of more fundamental, non-powerful 'categorical' properties. In contrast, on the dynamical operator account, powers are not only full-fledged members of our ontology, but also themselves perform a genuinely causal activity, one which we describe as functional in light of its similarity to the operation of mathematical functions. 
On the dynamical operator account of powers, what it is to be a power is to be that very activity. Because previous accounts of powers have attempted to derive the identity of those properties from what they can do in a productive sense, the causal-cum-modal complexity of their manifestations remains an inexplicable datum for which both unity and intrinsicality become insolubly problematic. ${ }^{22}$ On our novel account however, wherein the identity of powers consists in the activity which specifies how they do what they can do, the effects of a power are not identified with its essence but are rather its products - the former are the explanandum for which the latter is the explanans. Thus the unique causal role which we hold is the identity/essence of powers ensures that unity and intrinsicality are properly accounted for in a non ad hoc fashion: the nature of that role, as described above, provides a tangible sense in which a power's multiplicity of manifestations/effects cohesively belong to and metaphysically "flow from" its essence.

Of course, not just any collection of effects observed to be associated with a single entity will "flow from" the essence of a singular power it possesses. Our account is not meant to give conceptual justification for holding that every effect, or every counterfactual that might be associated with an entity must be metaphysically derivable from a single power's essence. As we mentioned in an earlier section, following this line of thought leads to accounts which license the formation of 'mega-powers' constructed from wildly disparate effects, a result which we consider both undesirable and untenable. ${ }^{23}$ In general, we believe that it is the purview of the empirical sciences to delineate which effects are to be associated with which powers; no plausible scientific understanding of $\mathrm{H} 2 \mathrm{O}$, for instance, will hold that its droplets forming spherical shapes on surfaces and its nourishing effects on plants are the causal resultants of a singular power. According to the dynamical operator account, the various manifestations of a power which can be captured in a series of qualitatively and quantitatively interrelated counterfactuals are metaphysically derivable from — and thus grounded in — a singular activity which constitutes that power's essence. But of course all but the very simplest entities will have multiple such powers, each with their own individual essence which performs a specific metaphysical mapping which causally 'translates' specific sets of stimulus factors into the production of a specific set manifestation states — and while our account does definitively say that will be the case it, nor any purely metaphysical account, cannot definitively assign which sets belong to which powers.

\footnotetext{
22 There have been attempts to address this challenge in the recent literature: in Handfield (2008) and Handfield (2010) the author suggests that the causal dynamics that a power participates in can provide a solution both to what we have called the 'unity problem' and the 'intrinsicality problem' (esp. in Handfield 2008: p. 121). However, while we claim there is a straightforward ontological distinction between a power and its manifestation, and we do not focus on the 'natural kinds' of manifestation processes (see also Ellis 2001: p. 162) in order to define what it is to be a power, according to Handfield (2008) it is only after having stipulated the existence of a pure causal process that we can define the power behind the process. Processes, however, involve a lot of elements, which all together converge to define what it is to be a power. In a sense, what we do is exactly the opposite: even if it is true that the manifestation of a disposition involves a causal process, and that the process needs to be of a certain type for it to be a manifestation of a particular disposition (see also Handfield, 2010), we try to focus on the activity of the power itself, excluding from the definition of the power the stimulus condition, the manifestation, and the whole spatiotemporal cause-effect process.
}

23 See also our remarks on 'qualitative multi-track' powers made in footnote 5. 
In the next, final section, we take a step backwards to put our new view of powers into context. We show how how our view differs importantly from the standard view with respect to the latter's central distinguishing features-namely, the way in which it characterises powers via the stimulus-manifestation model and its conception of the modality and directedness of powers.

\section{Dynamical operators: ontology and modality}

As described above, on our view, powers have a fundamentally dynamical essence-their very identity consists in their specialised activity. Powers, as we have said, are defined by what they do. Of course, this phrasing could mean two things. If we were to define say, blacksmiths, by "what they do", we plausibly could be signalling that their essence consists in their characteristic products-e.g. swords, shields, etc. Alternatively, we could instead be pointing to their characteristic activity-i.e. the very making of those products: the hammering, tempering, etc. of metals. These two ideas are obviously intimately related, but they are not identical: the latter indicates the intrinsic dynamical nature of 'the blacksmith', the former merely the extrinsic resultants of that nature. According to our account, it is the activity which has a place of primacy.

The aim of this section is to show why applying this line of thought to powers has important theoretical implications and signals a significant shift in the way powers have standardly been conceptualised. Most notably, our model challenges the standard model of powers. For from the metaphysics of powers we have proposed it follows that the stimuli and manifestations typically associated with powers must be relegated to occupying a purely epistemological and heuristic role, rather than an ontological and individuating one. In the contemporary literature the 'stimulus and manifestation' model, wherein what it is to be a particular power is equated with the counterfactual(s) that power makes-true, is nearly universally accepted. ${ }^{24}$ Consider 'negative charge'. A typical definition of the essence of this power via the stimulus-manifestation model will include a set of its characteristic manifestations - 'attracting other negative charges', 'repelling positive charges', 'generating an electromagnetic field', etc.

Putting the various problematic implications for it which we have discussed in the previous sections aside (with respect to extrinsicality, passivity, etc.), that model could plausibly be construed as one in which the essence of powers consists in what they do. However, on our view these manifestations, like the blacksmith's wares, are merely the dynamical consequences of the more fundamental activity of a power-namely, as we earlier detailed, its functioning as a dynamical operator which mediates the particular state-to-state value transitions which constitute all of its possible stimulusmanifestation pairs. This functional activity is what powers do-fundamentally — and hence on our account, constitutes their essence. It clearly follows then that in our

\footnotetext{
24 There are some notable exceptions however. Some authors place the manifestations alone-rather than the full counterfactuals - as the individuators of powers (Vetter 2015), while others abandon counterfactuals and opt for individuating powers via their 'causal influence' (Corry, 2019) or 'modal tendencies' (Mumford \& Anjum, 2011).
} 
model, in contrast to the standard stimulus-manifestation model, no collection of a power's characteristic manifestations can constitute its identity-conditions.

That said, while this collection has in our model lost its ontological import, because its members are the causal consequences of a power's essence it remains of significant epistemological and explanatory import: studying it can reveal heuristically valuable information that allows one to discover or further explicate that essence-e.g. cataloguing the electromagnetic interactions that occur between an electron and other particles (captured by a varied set of pleiotropic and polygenic counterfactuals) provides one with inferential insight into the dynamical activity of 'negative charge'. We acknowledge that on our metaphysical picture of the essence of powers there is a rather robust "epistemic gap" between our observations of a power's manifestations and its essence-the former we have immediate empirical access to while the latter must be inferentially "reconstructed" from the observational data of the former. We do not however view this as a cost of accepting our account as crossing this gap is a commonplace practice in the carrying-out of contemporary scientific investigation-e.g. the idealisation process that has led to the discovery of the laws of thermodynamics from the various empirical observations of the interactivity between 'energy', 'temperature' and 'entropy' in multiple distinct systems. We take our dynamical operator account to be the product of the same theoretical modus operandi in which the empirical sciences peer beyond the phenomenal veil of experience to discern the fundamental structure, relations, and laws that govern, or explain the state-to-state transitions of the physical world.

Our account's rejection of the standard model of powers has additional important implications for some of the core concepts associated with the metaphysics of powers-directedness and modality. Consider 'directedness' first. Directedness is a feature which has long been ascribed to powers, often in an attempt to ontologically distinguish those properties from so-called 'categorical' properties: powers are properties that are in some way "directed toward" the bringing about of particular possible, future states of affairs. ${ }^{25}$ That powers can be characterised by a form of 'physical intentionality'-a schema first offered by Place (1996) - has been controversial within the literature: some have argued that this model, taken from the philosophy of mind, is descriptively apt and gets at the essence of powers (Molnar, 2003; Mumford \& Anjum, 2011), while others have protested that the forward-looking nature of intentional states and their non-existent/unactualised objects have no place in one's ontology of the physical world-e.g. Armstrong's (1997) Menoingian objection to powers.

Putting this issue aside however, and accepting for the moment that powers are-in some sense-directed toward the production of particular states, the standard account of powers yet encounters a significant worry: given the pleiotropic and polygenic nature of powers (as we earlier detailed) one wonders precisely which manifestation is a particular power 'directed toward'? On the one hand, any account which claimed that a particular power is directed toward just one of the wide range of specific and particularised manifestations it is capable of must be seen as inadequate. On the other

\footnotetext{
25 See Cross (2005) for a good overview of the distinction between 'dispositional' and 'categorical' properties.
} 
hand, an account which claimed that a particular power is directed toward a 'determinable' manifestation (qua a generalised manifestation type) is implausible, ${ }^{26}$ given the non-fundamentality and idealised nature of determinables, especially with respect to causation. ${ }^{27}$ On the dynamical operator account of powers however, neither of these unattractive positions need be adopted, as powers are only 'directed toward' their manifestations in a rather weak and non-fundamental sense. According to that account, the intrinsic nature of powers relevant in causation is not their manifestations, but their functional activity - the former being only the extrinsically observable, dynamically derivative consequences of the latter. Thus our account does not need to make a declaration on which manifestation or set of manifestations any particular power is 'directed toward' for the simple reason that a power's manifestations do not directly represent its essence, but rather are causally contingent consequences of it. So long as 'directedness' is taken as a metaphysically innocuous description that highlights the dynamical results of a power, rather than as a fundamental, ontological feature of a power's essence, these problems can be avoided.

What implications does our downplaying the ontological significance of the directedness of powers have for the conception of them as modal properties? Typically, the directedness of powers is cashed-out via its truthmaking of counterfactuals. But as we have said that these counterfactuals are derivative and non-fundamental (and have accordingly relegated the directedness of powers to the same status) in what sense are our powers modal, given that this ascription generally depends upon such counterfactuals? Although on our account the set of counterfactuals which usually define the 'modal profile' of a power is not a part of its intrinsic nature, that does not entail that powers qua dynamical operators are not modal properties. In fact, we believe that on our account powers are more deservedly awarded the title of 'modal' than on the current standard, competitor accounts. Recall that on our account, every power-qua dynamical operator-is a particular kind of 'functional rule' whose activity converts input-states to output-states in a specific fashion. The ontological imposition of such rules in any causal context therefore metaphysically divides relevant pairs of state of affairs into ones which follow those rules and those which do not-e.g. the input-output pair $<$ striking, breaking $>$ follows the 'fragility' rule, but $<$ striking, floating $>$ does not. In this sense then, the activity of those operators define a set of pairs of states of affairs which are possible-i.e. which validly/coherently follow its specific dynamical rule.

Note that it is their performance of this metaphysical division which, as we earlier explained, allows powers to function as the ground of the 'dependence' and 'differencemaking' of causation insofar as this unique activity effectively generates the specific

\footnotetext{
26 Even if there are philosophers who have developed accounts that point in this direction: Rödl (2012) for example puts a lot of metaphysical weight on the 'determinable', and Mulder (2021) builds on Rödl's generic conception of powers to outline four varieties of powers (inanimate, animate, conscious and self-conscious). In these works we find something that can be linked to our present work (we thank an anonymous referee for this): a focus on the concept of 'dynamic activity' (what Rödl and Mulder call 'movement'); however, both in Rödl (2012) and in Mulder (2021), powers are 'general', in that they can be manifested in indefinitely many movements, and "powers are always powers for something: they specify a movement form, which in turn includes an end" (Mulder, 2021: p. 49). This is interesting but at odds with our purposes, as we try to exclude both the manifestation (even if 'general') and the 'end' from the definition of powers.

27 For a dissenting opinion on this matter, see Wilson (2012).
} 
sets of counterfactuals commonly associated with them. This is important because although those counterfactuals do not constitute the intrinsic nature of those powers-and thus, whatever 'modality' we assign to them cannot be straightforwardly assigned to powers - they are their direct dynamical products. Thus, powers qua dynamical operators serve as the metaphysical ground of the 'modal profile' commonly associated with powers. In other words, the functional activity which in our account constitutes the essence of powers is itself causally responsible for precisely the characteristics for which other accounts deem powers 'modal'. In our estimation this entails that the dynamical operator account-its conceptual relegating of the centrality of counterfactuals notwithstanding - posits powers which are in fact more deserving of the title 'modal properties' than its competitors.

\section{Concluding remarks}

In this paper, we have argued that powers are dynamical operators whose essence consists in the particular way in which they perform their functional activity of assigning state-to-state value transitions. After having introduced a common problem that many contemporary theories suffer from-compounding the causal complexity of powers, rather than accounting for it - we individuated two related desiderata that a promising account of powers should have: unity and intrinsicality. To properly satisfy unity, we have argued, it is necessary to satisfy the intrinsicality condition-and this is where the current theories are mostly deficient. In the second part of the paper we put forward a theory able to satisfy both desiderata: a new account of the essence of powers that focuses on their dynamical activity, rather then on the results brought about by this very activity. This, we argued, is a promising strategy to finally put powers in charge of the causal process and, in a sense, make powers actually powerful. Finally, we showed how this view differs from existing views with respect to the knowability of powers, their directedness and modality. We acknowledge that much work remains to be done on this new approach, but we believe that we have laid the foundation for that important future research here.

Open Access This article is licensed under a Creative Commons Attribution 4.0 International License, which permits use, sharing, adaptation, distribution and reproduction in any medium or format, as long as you give appropriate credit to the original author(s) and the source, provide a link to the Creative Commons licence, and indicate if changes were made. The images or other third party material in this article are included in the article's Creative Commons licence, unless indicated otherwise in a credit line to the material. If material is not included in the article's Creative Commons licence and your intended use is not permitted by statutory regulation or exceeds the permitted use, you will need to obtain permission directly from the copyright holder. To view a copy of this licence, visit http://creativecommons.org/licenses/by/4.0/.

\section{References}

Armstrong, D. (1997). A world of states of affairs. Cambridge University Press.

Beebee, H., Hitchcock, C., \& Menzies, P. (Eds.). (2009). The oxford handbook of causation. Oxford University Press.

Austin, C. (2015). The Dispositional Genome: Primus Inter Pares. Biology and Philosophy, 30(2). 
Austin, C. (2018). Essence in the age of evolution: A new theory of natural kinds. Routledge.

Bird, A. (1998). Dispositions and antidotes. The Philosophical Quarterly, 48(191), 227-234.

Bird, A. (2007). Nature's metaphysics: Laws and properties. Oxford University Press.

Boyd, R. (1999). Homeostasis, species, and higher taxa. In R. Wilson (Ed.), Species: New interdisciplinary essays (pp. 141-186). The MIT Press.

Carnap, R. (1936). Testability and meaning. Philosophy of Science, III, 419-471.

Chakravartty, A. (2007). A metaphysics for scientific realism: Knowing the unobservable. Cambridge University Press.

Choi, S. (2006). The Simple vs. reformed conditional analysis of dispositions. Synthese, pp. 369-379.

Corry, R. (2019). Power and influence: The metaphysics of reductive explanation. Oxford University Press.

Cotnoir, A., \& Baxter, D. (2014). Composition as Identity. Oxford University Press.

Cross, T. (2005). What is a disposition? Synthese, pp. 321-341.

Dumsday, T. (2019). Dispositionalism and the metaphysics of science. Cambridge University Press.

Ellis, B. (2001). Scientific essentialism. Cambridge University Press.

Ellis, B. (2002). The philosophy of nature: A guide to the new essentialism. Mcgill-Queen's University Press.

Froeyman, A. (2012). The ontology of causal process theories. Philosophia, 40(3), 523-538.

Glennan, S. (1996). Mechanisms and the nature of causation. Erkenntnis, 44(1), 49-71.

Glynn, L. (2013). Causal foundationalism, physical causation, and difference-making. Synthese, 190(6), 1017-1037.

Goodman, N. (1954/1979). Fact, fiction, and forecast. Cambridge: Harvard University Pres.

Gundersen, L. (2002). In defence of the conditional account of dispositions. Synthese, pp. 389-411.

Handfield, T. (2008). Humean dispositionalism. Australasian Journal ofPhilosophy, 86, 113-126.

Handfield, T. (2010). Dispositions, manifestations and causal structure. In A. Marmodoro (Ed.), The metaphysics of powers - Their grounding and their manifestations (chap. 7). New York: Routledge.

Hauska, J. (2009). Dispositions unmasked. Theoria, 75(4), 304-335.

Heil, J. (2003). From an ontological point of view. Clarendon Press.

Heil, J., \& Martin, C. (1999). The ontological turn. Midwest Studies in Philosophy, pp. 34-60.

Johnston, M. (1992). How to speak of the colors. Philosophical Studies, pp. 221-263.

Keller, P. (2007). A world of truthmakers. In J.-M. Monnoyer (Ed.), Metaphysics and truthmakers (pp. 105-156). Ontos Verlag.

Lewis, D. (1973). Causation. Journal of Philosophy, pp. 556-567.

Lewis, D. (1986). On the plurality of worlds. Basil Blackwell.

Lewis, D. (1997). Finkish Dispositions. The Philosophical Quarterly, pp. 143-158.

Lowe, E. (2006). The four-category ontology: A metaphysical foundation for natural science. Clarendon Press.

Lowe, E. J. (2010). On the individuation of powers. In A. Marmodoro (Ed.), The metaphysics of power$s$-Their grounding and their manifestations (pp. 8-26). Routledge.

Manley, D., \& Wasserman, R. (2008). On linking dispositions and conditionals. Mind, pp. 59-84.

Martin, C. (1994). Dispositions and conditionals. The Philosophical Quarterly, pp. 1-8.

Martin, C. (2007). The mind in nature. Oxford University Press.

Mellor, D. (1974). In defense of dispositions. The Philosophical Review, pp. 157-181.

Molnar, G. (2003). Powers: A study in metaphysics. Oxford University Press.

Mulder, J. (2021). Varieties of powers. Axiomathes, 31, 45-61.

Mumford, S. (2004). Laws in nature. Routledge.

Mumford, S., \& Anjum, R. (2011). Getting causes from powers. Oxford University Press.

Oderberg, D. (2011). Essence and properties. Erkenntnis, 75(1), 85-111.

Place, U. (1996). Intentionality as the mark of the dispositional. Dialectica, 50(2), 91-120.

Psillos, S. (2004). A glimpse of the secret connexion: harmonizing mechanisms with counterfactuals. Perspectives on Science, 12(3), 288-319.

Rödl, S. (2012). Categories of the temporal. Harvard University Press.

Ryle, G. (1949). The concept of mind. Hutchinson \& Co.

Schrenk, M. (2010). The Powerlessness of Necessity. Nous, 44(4), 725-739.

Stalnaker, R. (1968). A Theory of Conditionals. Studies in Logical Theory. American Philosophical Quarterly Monograph Series, pp. 98-112.

van Inwagen, P. (1990). Material Beings. Cornell University Press.

Vetter, B. (2013). Multi-track dispositions. The Philosophical Quarterly, 63, 330-352. 
Vetter, B. (2015). Potentiality: From dispositions to modality. Oxford University Press.

Williams, N. (2011). Putting powers back on multi-track. Philosophia, 39(3), 581-595.

Williams, N. (2019). The powers metaphysic. Oxford University Press.

Wilson, J. (2012). Fundamental determinables. Philosopher's Imprint, 12(4), 1-17.

Wilson, R., Barker, M., \& Brigandt, I. (2007). When traditional essentialism fails: Biological natural kinds. Philosophical Topics, 35(1/2), 189-215.

Woodward, J. (2003). Making things happen: A theory of causal explanation. Oxford University Press.

Publisher's Note Springer Nature remains neutral with regard to jurisdictional claims in published maps and institutional affiliations. 